\title{
Using Fuzzy Inference system for detection the edges of Musculoskeletal Ultrasound Images
}

\author{
Shaima I. Jabbar Member, IEEE, Charles R. Day, and Edward K. Chadwick
}

\begin{abstract}
Edge detection in Musculoskeletal Ultrasound Imaging readily allows an ultrasound image to be rendered as a binary image. This facilitates automated measurement of geometric parameters, such as muscle thickness, circumference and cross-sectional area of the tendon. In this work, we introduced a new method of edge detection based on a fuzzy inference system and apply it to the ultrasound image. An anisotropic diffusion filter was used to reduce speckle noise before implementation of the edge detection method, which consists of three characteristic steps. The first step entailed fuzzification, for which three fuzzy membership functions were applied to the image. The parameters of these functions were selected based on an analysis of the standard deviation of grey level intensities in the image. Secondly, 12 fuzzy rules for identifying edges were constructed. Thirdly, defuzzification was carried out using the Takagi-Sugeno method. Furthermore, a reference-based edge measurement was quantitatively determined by comparing edge characteristics with a standard reference. We made two inferences from our observations. Firstly, the ability to automatically identify the important details of a musculoskeletal ultrasound image in a very short time is possible. Secondly, this method is effective compared with other methods.
\end{abstract}

Index Terms-Edge detection, Fuzzy inference technique, Musculoskeletal Ultrasound imaging, Musculoskeletal Modelling

S. I. Jabbar, Al Furat Al Awsat Technical University, Iraq (shaima.jabbar18@gmail.com) and IEEE member (jabbar.s.i@ieee.org).

C.R. Day, Keele University, UK (c.r.day@keele.ac.uk).

E. K. Chadwick, Institute for Science and Technology in Medicine Keele University, UK (e.k.j.chadwick@keele.ac.uk). This work was supported financially by Iraqi government, Ministry of Higher Education and Scientific Research.

\section{INTRODUCTION}

Ultrasonography has previously been used for manual labelling of geometric parameters of the musculoskeletal system [1][2], but it has not yet accurately identified the shape and size of the musculoskeletal components. Added to that, it is very time-consuming. An automated interpretation aimed at extracting geometric parameters based on the analysis of the image properties efficiently and rapidly. Segmentation of the musculoskeletal ultrasound image is often the main objective of geometric parameter extraction of the musculoskeletal system. Edge detection is a prerequisite for image segmentation because it typically allows the image to be represented by two colours (black and white) [3]. The primary purpose of edge detection is the identification of the shape and size of an object in the image, such as a muscle or tendon. An improved edge detection method is likely to be a valuable tool for several applications, such as general computer vision and for geometric parameter detection methods using Hough transforms. The Hough transform method has been used in line or circle identification [4]. Several different methods for edge detection have been utilised on musculoskeletal US images. One of the preliminary works in musculoskeletal US image segmentation was introduced by Gupta [5]. This work was based on curvelet transforms and morphological image processing (dilation and erosion). Moreover, speckle noise was dealt with by the statistical adaptive method and an anisotropic diffusion filter before going on to perform musculoskeletal US image segmentation. Other approaches have attempted edge detection of panoramic musculoskeletal ultrasound images [6] and X-ray images of bone [7] by using Convolutional Neural Networks (CNNs). The CNN technique can be time-consuming because it needs extensive training and large training datasets to get a robust edge detection output image. In CNN approaches, the image is typically processed without addressing the low contrast and speckle noise problems to assess the ability of CNNs to perform edge detection without image preprocessing. Furthermore, edge detection methods based on fuzzy logic techniques were previously applied on images but not medical images [8], [9], [10] and medical image [11], [12], [13]. However, in these techniques, several grey level colors have been detected in the output image. This complicates the complete segmentation an object from other image details (e.g. the separation of a tendon or a muscle from its background in the musculoskeletal ultrasound image).

In many of the above approaches, the details of edges in a resultant image are highly interconnected. Effectively, this means we may see the edges of the muscle boundaries or tendon, bone and other details together in one image. If the purpose was to measure only muscle borders, however, then only the edges of muscle borders are highly desirable, and it would be crucial to exclude other details and thus minimise artefacts. This would allow us to optimise edge detection for the structures of interest and reject those not required. In this regard, the specificity and quality of generated edges exert a large impact on the accuracy of automatic analysis of musculoskeletal components.

In this work, we introduced a new method of edge detection based on a fuzzy technique, knonwn as the Fuzzy Edge Detection Method (FEDM). This approach depends on a Fuzzy Inference System (FIS). It is conducted by fuzzification of the image with the optimal selection of the fuzzy membership functions, construction of fuzzy rules for the edge detection in the fuzzy domain, and finally defuzzification. The selection of parameters for membership functions relies on the analysis of the standard deviation curve of a set of thresholded images because this curve depicts the variation of grey level intensities. The fuzzy rules reflect the knowledge of representation and detection of the enhanced image's edges. Different levels of information can be deduced using this method. It is possible to exclude unnecessary information (e.g. texture or speckle information) and keep the most appropriate edge detection 
information. Moreover, FEDM is relatively simple and completely automatic. Its primary advantage is that it is a robust edge detection method that imparts a clear description of the object's properties inside the musculoskeletal US images. However, there is a relationship between a successful method of edge detection and the accuracy of the automatic measurement.

The remainder of this paper is organised as follows: a brief review of related measurement metrics in the same section, FEDM (methods) is explained in detail in section II. Experimental results are shown in section III, followed by discussion in section IV. The final section contains conclusions and future work.

\section{Metrics of Edge Measurement based on Reference image}

Measurement of the FEDM's performance gives us evidence about the technique's effectiveness and helps in improving edge detection method to get a high level of accuracy. The edge map can be assessed using subjective and objective evaluations. Subjective evaluation can be carried out based on observers' opinions and rating scales such as Mean Opinion Score (MOS), while objective evaluation is determined based on comparing the resultant edge image to a reference image. Often, a reference-based, objective measurement is recommended because MOS might be biased in the visual interpretation and it is difficult to measure the quality of edge performance without using a reference image [14]. Reference image reflects the expertise of the expert in demonstrating the most important details in the image to create this image. Different statistical methods have been used in reference objective assessment; some of them are F-measure and MCC (Matthews Correlation Coefficient). These methods include extraction TP (True Positive), FP (False Positive), TN (True Negative) and FN (False Negative) by matching pixels of detected edge image and reference image [15]. Although an expert has the experience to trace the important details of the input image, it is not possible to say that reference image is faultless in assigning the precise location of the pixel; because the expert will draw it manually. Therefore, comparison between the detected image and ground truth image based on a pixel-by-pixel assessment may not give us an accurate evaluation. However, if the match between edge maps of detected and ground truth images is tested by analysis of higherlevel edge characteristics, it might avoid the pitfalls of a pixel by pixel comparison. Analysis of edge characteristics might include evaluations such as distance between actual edge and ideal edge pixel, thick edge occurrence and edge pixel presence. The first standard method, which was used in this matching, was Pratt's Figure of Merit (FOM) method; it is shown in equation (1).

$$
F O M_{\text {Pratt }}=\frac{1}{\max \left\{N_{g}, N_{a}\right\}} \sum_{i=1}^{N_{a}} \frac{1}{1+\propto d^{2}}
$$

where $N_{g}$ is number of edge pixel in ground truth image, $N_{a}$ is number of actual edge pixel, $d$ is distance between actual edge pixel and the nearest ideal edge pixel and $\alpha$ equals $1 / 9$ (at this value, a reasonable edge position is detected, suggested by Pratt) [16]. Moreover, the improved version of this method was achieved by Pinho for giving more effective accounting of false edges, as shown in equation (2).

$$
\text { Pinho }=\left[\frac{1}{N_{g}} \sum_{k=0}^{N_{g}} \frac{1}{1+\propto d^{2}}\right]\left[\frac{1}{1+\beta \frac{N_{F P}}{N_{g}}}\right]
$$

where $N_{F P}$ is number of false positive pixels and the value of $\beta$ is 1 , but in the case of $N_{F P}=N_{g}$, the value of this parameter will be 0.5 [17]. Recently, Reference-Based Edge Measure (RBEM) has been introduced and applied to simple and natural images. In this metric, four edge characteristics are fused together: evaluation of edge connectivity, thick edge occurrence, edge localization and edge corner presence, to get an improved level of the quality assessment. This method demonstrated a high agreement with subjective assessment, which has been estimated by collecting opinions of experts (MOS). Equation (3) presents the main components of RBEM.

$$
\begin{gathered}
R B E M=\psi_{L}\left(1-D_{L}\right)+\psi_{C O}\left(1-D_{C O}\right)+\psi_{T H}\left(1-D_{T H}\right) \\
+\psi_{S}\left(1-D_{S}\right)
\end{gathered}
$$

where $D_{L}$ is measurement of the edge pixels localization, $D_{C O}$ is to measure edge corner presence, $D_{T H}$ demonstrates the thick edge occurrence measurement and $D_{S}$ is measurement of isolated pixels in output edge detection image. $\Psi_{L}, \Psi_{C O}, \Psi_{T H}$ and $\Psi_{S}$ are parameters for edge pixel, edge corner, thickness of edge and isolated pixels respectively. The values of these parameters used in the natural images were $\Psi_{L}=0.63, \Psi_{C O}=-0.02, \Psi_{T H}$ $=1.35$ and $\Psi_{S}=-1.28$. All details of determination $D_{L}, D_{C O}$, and $D_{S}$ are as suggested in [18] and [14].

\section{FUZZY EDGE DETECTION METHOD (FEDM).}

This technique is based on a fuzzy inference system and composed of the following steps: recruiting membership functions are defined by selection of the appropriate parameters (fuzzification) in the first step. The second step is the construction of fuzzy rules that reflect a simple representation of the edge pixels as $3 \times 3$ mask, and the last step is defuzzification (see Fig.1 below).

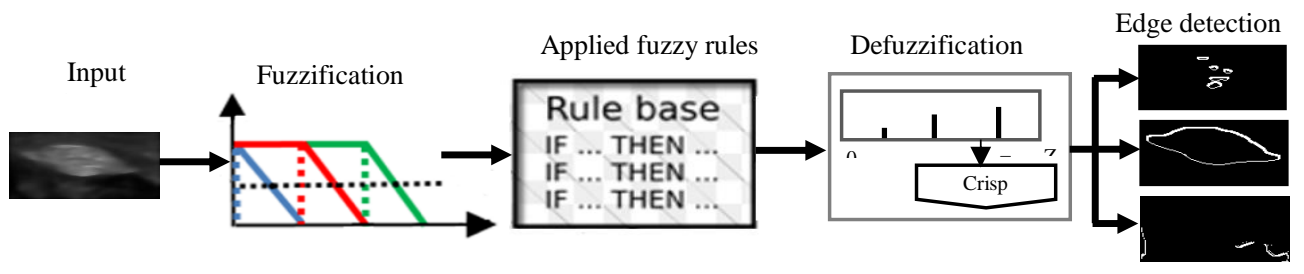

Fig. 1 shows the pipeline of FEDM structure 


\section{a. Fuzzification}

The purpose of fuzzification is to map the grey level intensities of the image from the spatial domain to the fuzzy domain. Fuzzification can be done by choosing a suitable membership function [19]. The two most important aspects of the membership function are its form and the parameters that described the behaviour of these functions. In this work, we established three membership functions for three different levels of edge detection information. Parameters of these functions were selected according to the analysis of image properties. Assuming $(i, j)$ are the spatial coordinates of each pixel in the input image $I$ of size NxM, gmax is the maximum grey level intensity in the input image and gmin is the minimum, $I(i, j) \in[$ gmin, gmax $]$. The intensity of the input image (spatial domain) is mapped to the interval [0,1] (fuzzy domain), equation (4) gives us a general aspect of these functions.

$$
\mu_{a, C p_{a-1}, C p_{a}}(i, j)=\operatorname{mapping}(I(i, j))
$$

where multi membership functions are $\mu_{a, C p_{a-1}, C p_{a}}(i, j)[0,1]$, $a=1,3$, and $\left(C p_{a-1}, C p_{a}\right)$ are parameters of $\mu_{a}(i, j)$.

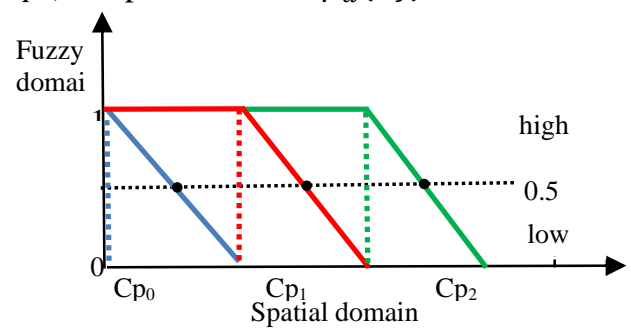

Fig.2 illustrates three membership functions $\mu_{1}(i, j), \mu_{2}(i, j)$ and $\mu_{3}(i, j)$ in different colors, blue for first membership function in the case of level 1 , red for level 2 and green color for representation of the membership function of level 3. Where, $\left(\mathrm{Cp}_{0}, \mathrm{Cp}_{1}, \mathrm{Cp}_{2}\right.$, and $\left.\mathrm{Cp}_{3}\right)$ are parameters of these membership functions.

Fig. 2 shows the form of the three membership functions in different colors; this illustrates the mapping of grey level intensities from the spatial domain to the fuzzy domain for three different levels of edge detection information. High membership values in all three different levels are greater or equal 0.5 , while low values are under 0.5 .

The important question is, how can a good set of membership functions be constructed for musculoskeletal ultrasound image domain using parameters $\left(\mathrm{Cp}_{0}, \mathrm{Cp}_{1}, \mathrm{Cp}_{2}\right.$, and $\left.\mathrm{Cp}_{3}\right)$ ? In this work, we introduce a new method for the selection of these parameters. This method relies on an analysis of the standard deviation of grey level intensities instead of looking at the grey level intensities themselves, or analysis of the shape of the image histogram. Most medical images, particularly musculoskeletal US images, have heterogeneous regions of grey level intensities; this leads to difficulties in interpreting the shape of the histogram due to its many peaks and valleys [20]. Standard Deviation (SD) of grey level intensity provides a simple summary measure of the amount of data variability in the image. Before the standard deviation of the intensities is evaluated the original image is thresholded several times across a range of thresholds using equation (5):

$B_{h}(i, j)= \begin{cases}I(i, j) & \text { if } I(i, j) \geq T_{h} \\ 0 & \text { else }\end{cases}$ where $I(i, j)$ is the original input image with dimension $\mathrm{i}$ and $\mathrm{j}$, $h=1,2 \ldots . . n, h$ :subscript for each of grey scale threshold that are applied to the original image. $T_{h}$ is the threshold used to generate the output image $B_{h}(i, j)$ and $T_{h}$ takes integer values over the range [gmin, gmax]. Based on the SD values, which are calculated for each $B_{h}(i, j)$ images, an SD curve can be plotted against cut-off intensity, see figure (3). The SD curve gives us an indication of the variation of grey level intensities. If the cutoff point $=$ gmin, this means the SD will be calculated for the original image, and if the image has a low SD value, there less variability in the grey level intensities as compared to a high level of SD. Furthermore, the complexity and amount of computation will be increased as the size of the $i$ and $j$ increase (approximately $\mathrm{O}\left(\mathrm{n}^{2}\right)$ in the size of the image). A novel aspect of our proposed fuzzy edge detection method is to exploit the characteristic shape (see figure 3 , below) of this SD curve for thresholded musculoskeletal US images, which arises to $\mathrm{SD}_{\max }$ before falling away.

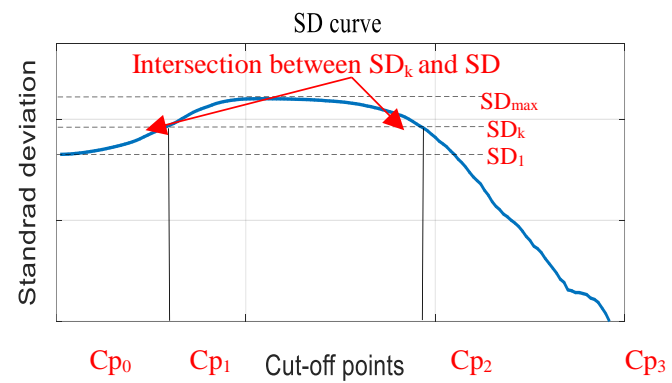

Fig.3, illustrated plot of SD curve which calculated from a set of SD values. In this curve the value of $\mathrm{SD}_{\mathrm{k}}$ is calculated at $\mathrm{m}_{\mathrm{k}}=0.5$ as example.

By collecting together SD values for each thresholded image they can be used to determine four parameters of the fuzzy membership functions, as follows:

1- Extract a set of the thresholded images based on a set of cut-off points with the range [gmin, gmax] by using equation (5), to get a set of thresholded images $B_{h}(i, j)$, $h=1,2, \ldots . n$.

2- $\quad$ For each thresholded image $B_{h}(i, j)$, calculate and save the standard deviation of pixel intensities, in order to get $S D_{1}\left(B_{1}(i, j)\right), S D_{2}\left(B_{2}(i, j)\right), S D_{3}\left(B_{3}(i, j)\right) \ldots S D_{n}\left(B_{n}(i, j)\right)$.

3- Assign $\left(S D_{l}\right)$ as the value of SD at initial state (at first cutoff point) and find $S D_{\max }$, which represents the maximum value of the SD set.

4- Calculate $S D_{k}$ using equation (6):

$S D_{k}=m_{k}\left(S D_{\max }-S D_{1}\right)+S D_{1}$

where $m_{k}$ is control parameter, $\mathrm{k}=1,2, \ldots 10$ and $m_{l=0.1 \text {, }}$ $m_{2}=0.2 \ldots m_{10}=1$.

5- This step consists of three parts as follows: -

a. Calculate the difference between $S D_{k}$ and the array of $\mathrm{SD}$ values and save the result.

b. Detect the minimum differences which belong to cutoff points and save these cut-off points. Where, minimum difference points represent the intersection between $\mathrm{SD}_{\mathrm{k}}$ and the SD array (as illustrated by Fig.3). 
c. Translate these cut-off points to the grey level intensities to get $\left(\mathrm{Cp}_{1}\right.$ and $\left.\mathrm{Cp}_{2}\right)$.

6- Add one row of the matrix at recording membership parameters $\left(\mathrm{Cp}_{0}, \mathrm{Cp}_{1}, \mathrm{Cp}_{2}\right.$ and $\left.\mathrm{Cp}_{3}\right)$, these parameters defining fuzzy membership function in Fig.3. Where, $\left(\mathrm{Cp}_{1}, \mathrm{Cp}_{2}\right)$ are obtained from the previous step and $\left(\mathrm{Cp}_{0}\right.$, $\mathrm{Cp}_{3}$ ) represents gmin and gmax respectively. Change the parameters of each row of the matrix depends on the changing the $m_{k}$ value, go back to the step 4 .

7- For each row of this matrix calculate $\mathrm{RBEM}_{\mathrm{k}}$ to find the best value of $m_{k}$.

where $\left(\mathrm{Cp}_{0}, \mathrm{Cp} \mathrm{p}_{1}\right)$ are parameters of the fuzzy membership function of Level $1,\left(\mathrm{Cp}_{1}, \mathrm{Cp}_{2}\right)$ are parameters of the fuzzy membership function of Level 2 and $\left(\mathrm{Cp}_{2}, \mathrm{Cp}_{3}\right)$ are parameters of the fuzzy membership function of Level 3.

\section{b. Applied fuzzy rules}

As shown in Fig. 4, where $\mathrm{C}_{5}$ is the particular pixel in $\mu_{a}(i, j)$ and $\left(\mathrm{C}_{1}, \mathrm{C}_{2}, \mathrm{C}_{3}, \mathrm{C}_{4}, \mathrm{C}_{6}, \mathrm{C}_{7}, \mathrm{C}_{8}, \mathrm{C}_{9}\right)$ are neighbour pixels of the $\mathrm{C} 5$ pixel. The black color in $3 \times 3$ mask represent high probabilities of dark grey level intensities, while the white color reflects high probabilities of bright grey level intensities.

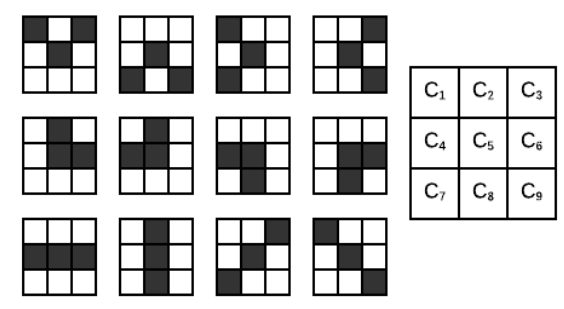

Fig. 4, mask configuration for detection image's edges.

Twelve fuzzy rules were introduced to represent different types of edge pixel in a $3 \times 3$ mask, which was moved across the whole image. If the weights of central pixel and two neighbor pixels are high degree of membership (fuzzy set is greater than or equal to 0.5 ) and the weights of remaining five neighbour pixels are low degree (fuzzy set is less than 0.5), then the central pixel represents an edge, see Fig.3. Rules are demonstrated as the following: -

Rule 1: If $\left(\mathrm{C}_{1}, \mathrm{C}_{3}, \& \mathrm{C}_{5}\right)$ are high \& $\left(\mathrm{C}_{2}, \mathrm{C}_{4}, \mathrm{C}_{6}, \mathrm{C}_{7}, \mathrm{C}_{8}, \& \mathrm{C}_{9}\right)$ are low then central pixel is edge.

Rule 2: If $\left(\mathrm{C}_{5}, \mathrm{C}_{7}, \& \mathrm{C}_{9}\right)$ are high \& $\left(\mathrm{C}_{1}, \mathrm{C}_{2}, \mathrm{C}_{3}, \mathrm{C}_{4}, \mathrm{C}_{6}, \& \mathrm{C}_{8}\right)$ are low then central pixel is edge.

$\cdots$

Rule 12: If $\left(\mathrm{C}_{1}, \mathrm{C}_{5}, \& \mathrm{C}_{9}\right)$ are high \& $\left(\mathrm{C}_{2}, \mathrm{C}_{3}, \mathrm{C}_{4}, \mathrm{C}_{6}, \mathrm{C}_{7}, \& \mathrm{C}_{8}\right)$ are low then central pixel is edge.

\section{c. Defuzzification}

Defuzzification is concerned with transforming the information from the fuzzy domain to the spatial domain to obtain the output image. Different defuzzification methods can achieve this, such as mean of maximum method, center of gravity method and using inverse membership function [21]. In this work, the Takagi and Sugeno method [22] is selected to detect output edges, each rule has a crisp output, and the final output is evaluated by weighting the average of rules using equation (7):

$$
\text { Final output }=\sum_{d=1}^{R} W_{d} Z_{d} / \sum_{d=1}^{R} W_{d}
$$

where, $W_{d}$ is rule weight (antecedent), the part of the sentence after IF in the rule, while $Z_{d}$ represents output level (consequent or conclusion in the rule) and $d=1 \ldots R, R$ is number of rules and equal 12. This method was designed to get an edge detection image with two colors (black and white) and is not time consuming. Ultimately, different levels of edge detection images were extracted. It is possible to choose the most appropriate level of information and exclude unnecessary regions to get a powerful final edge detection image. However, it is useful to additionally remove some individual pixels or thin double layers of edges from the final edge detection image using morphological operations [23].

\section{EXPERIMENTAL RESULTS}

In this section, proposed method was applied on two experiments (experiment1 and experiment2). A LOGIQ S7 ultrasound machine was utilized at $12 \mathrm{MHz}$ and a static scanning technique employed to obtain a single snapshot. 25 image samples were collected in the experiment1, while 20 sampled in the experiment 2. Furthermore, ground truth images for these samples in both experiements were drawn by an expert, who concentrated on identifying the boundaries of the cross section of the flexor pollicis longus tendon in the in the experiment 1 and triceps muscle boarders in the experiment 2 .

In this work, the pipelines of proposed method consist of several steps: denoising MUI using anisotropic diffusion filter [24], [25], [26], plotting SD curve, extraction parameters of fuzzy membership function based on SD curve. After that, fuzzification, applying fuzzy rules and defuzzification were carried out.

Appropriate parameters for membership functions $\left(\mathrm{Cp}_{0}, \mathrm{Cp}\right.$, $\mathrm{Cp}_{2}$, and $\mathrm{Cp}_{3}$ ) must be selected before fuzzification. These parameters were selected according to the SD curve. The SD curve is formed by determining SD of a set of thresholded images, the range of cut-off points between minimum and maximum values of the image. Extraction of $S D_{1}$ and $S D_{\max }$ from SD curve was achieved (as described in section II, fuzzification). $S D_{k}$ was calculated using equation (6) at different values of $m_{k}$; a set of parameters $\left(\mathrm{Cp}_{0}, \mathrm{Cp}_{1}, \mathrm{Cp}_{2}\right.$, and $\left.\mathrm{Cp}_{3}\right)$ were extracted at every change of $m_{k}$ value, so we can generate a matrix, which includes this information. Each row of this matrix illustrates different parameters of the membership function. Selection of the best value of $m_{k}$ depends on the evaluation of $\mathrm{RBEM}_{\mathrm{k}}\left(\mathrm{RBEM}_{\mathrm{k}}\right.$ is calculated using equation (3)), the best value of $m_{k}$ will be chosen at high score of RBEM.

Twelve fuzzy rules (see section II, applied fuzzy rule) were applied to the image of sample 1 (see table I) after implementing fuzzification stage and construction of three membership functions (see Fig.2). Three levels of the edge detection information were obtained after defuzzification using equation (7); an edge detection image, which includes necessary information will be kept, and pixel information from other levels such as texture or sporadic pixels will be rejected. Sometimes it is necessary to utilize post-processing such as morphological operations (thinning) [23]. This is used as a post- 
processing step to remove isolated pixels or to get single pixel thickness layer in the final edge detection image. RBEM was employed to do assessment between the final edge detection image and ground truth image. The value of $S D_{k}$ controls the decision of all levels of information because the intersection between $\mathrm{SD}_{\mathrm{k}}$ and $\mathrm{SD}$ curve determined the parameter values $\left(\mathrm{Cp}_{1}, \mathrm{Cp}_{2}\right)$. Table I, illustrates the steps of implantation proposed method at experiment 1 and experiment 2 then compared the results with other method using Canny edge detection operator.

\begin{tabular}{|c|c|c|}
\hline Table I & & \\
\hline Steps & Experiment 1 & Experiment 2 \\
\hline $\begin{array}{l}\text { Data } \\
\text { collection }\end{array}$ & $\begin{array}{l}\text { Data was collected from } 25 \text { healthy volunteers (9 } \\
\text { females and } 16 \text { males), age } 39 \pm 15 \text { years. All } \\
\text { participants provided written informed consent in } \\
\text { accordance with institutional ethical guidelines } \\
\text { (Ref No: ERP1290). This data came from } \\
\text { transverse scanning of the right and left-hand, in } \\
\text { the regions between the carpometacarpal joint and } \\
\text { the metacarpophalangeal joint. }\end{array}$ & $\begin{array}{l}\text { Data was collected from } 20 \text { healthy volunteers (4 } \\
\text { females and } 16 \text { males), as the same ethics of } \\
\text { experiment } 1 \text {. Single snapshot of the ultrasound static } \\
\text { scanning to identify triceps brachii muscle, the position } \\
\text { of the volunteer participant is opposite to the researcher } \\
\text { who works on ultrasound machine; elbow joint of the } \\
\text { volunteer flexed } 90^{\circ} \text { with the palm resitting on the table. }\end{array}$ \\
\hline $\begin{array}{l}\text { Speckle } \\
\text { noise } \\
\text { reduction }\end{array}$ & $\begin{array}{l}\text { An anisotropic diffusion filter was administered to } 25 \text { samples } \\
\text { of the healthy image to reduce speckle noise. This is sample } 1 \\
\text { after and before applying this filter is presented as example } \\
\text { below. }\end{array}$ & $\begin{array}{l}\text { The same filter which used in the experiment } 1 \text {, it applied on } 20 \\
\text { samples in this experiment, example is shown below: } \\
\text { Input image }\end{array}$ \\
\hline $\begin{array}{l}\text { Plot SD } \\
\text { curve }\end{array}$ & $\begin{array}{l}\text { The SD curve is formed by determining } \mathrm{SD} \text { of a set of } \\
\text { thresholded images, the range of cut-off points between } \\
\text { minimum and maximum values of the image. }\end{array}$ & $\begin{array}{l}\text { Following the same procedure in the experiment } 1 \text { to extract } \mathrm{SD} \\
\text { curve. }\end{array}$ \\
\hline $\begin{array}{l}\text { Parameters } \\
\text { extraction } \\
\text { of } \\
\text { membership } \\
\text { function } \\
\text { based on } \\
\text { SD curve }\end{array}$ & $\begin{array}{l}\text { This figure shows selection the best value of } m_{k} \text { based on } \\
\mathrm{RBEM} \text { evaluation to calculate } S D_{k} \text { and then get the proper } \\
\text { parameters }\left(\mathrm{Cp}_{1}, \mathrm{Cp}_{2}\right) \text { of the membership functions in the case } \\
\text { of sample } 1 \text {. The best value of } m_{k} \text { is } 0.1 \text { at } \mathrm{RBEM}_{\mathrm{k}}=0.621 \text {. }\end{array}$ & (ligh scorr of \\
\hline $\begin{array}{l}\text { Applied } \\
\text { FEDM on } \\
\text { MUI }\end{array}$ & Level 3 & $\frac{\text { Level2 }}{-\mathrm{ssc}^{2}}$ \\
\hline $\begin{array}{l}\text { Comparison } \\
\text { with Canny } \\
\text { edge } \\
\text { detection } \\
\text { method }\end{array}$ & Proposed image & Canny method \\
\hline
\end{tabular}


The same procedure was done on the rest of 25 healthy image samples. To evaluate fuzzy edge detection method performance, Pratt's FOM, Pinho and RBEM were employed. Furthermore, fuzzy edge detector was replaced with Canny edge detector [3]; the same metrics were employed on it to see performance difference between each two methods. Table II demonstrates the quantitative assessment of all healthy samples (25 images) using fuzzy and Canny edge detector. High scores in these metrics denote high performance for the method.

\begin{tabular}{|c|c|c|c|c|c|c|c|c|c|c|c|c|}
\hline \multirow{3}{*}{$\mathrm{N}$} & \multicolumn{6}{|c|}{ Experiment 1} & \multicolumn{6}{|c|}{ Experiment 2} \\
\hline & \multicolumn{3}{|c|}{ FEDM } & \multicolumn{3}{|c|}{ Canny } & \multicolumn{3}{|c|}{ FEDM } & \multicolumn{3}{|c|}{ Canny } \\
\hline & RBEM & FOM & Pinho & FOM & RBEM & Pinho & RBEM & FOM & Pinho & FOM & RBEM & Pinho \\
\hline 1 & 0.621 & 0.653 & 0.500 & 0.600 & 0.192 & 0.245 & 0.6092 & 0.5423 & 0.3902 & 0.6086 & 0.0672 & 0.1091 \\
\hline 2 & 0.618 & 0.540 & 0.418 & 0.600 & 0.400 & 0.300 & 0.6251 & 0.3343 & 0.2461 & 0.614 & 0.0991 & 0.1458 \\
\hline 3 & 0.604 & 0.500 & 0.407 & 0.600 & 0.401 & 0.370 & 0.6140 & 0.4120 & 0.3050 & 0.6244 & 0.0959 & 0.1373 \\
\hline 4 & 0.616 & 0.540 & 0.402 & 0.615 & 0.273 & 0.300 & 0.6079 & 0.4596 & 0.3440 & 0.6141 & 0.0894 & 0.1358 \\
\hline 5 & 0.615 & 0.51 & 0.402 & 0.609 & 0.390 & 0.333 & 0.6133 & 0.3385 & 0.2537 & 0.6176 & 0.0872 & 0.1344 \\
\hline 6 & 0.606 & 0.47 & 0.360 & 0.600 & 0.233 & 0.265 & 0.6202 & 0.3235 & 0.2375 & 0.6121 & 0.1028 & 0.1462 \\
\hline 7 & 0.620 & 0.609 & 0.447 & 0.604 & 0.290 & 0.296 & 0.6200 & 0.2706 & 0.2257 & 0.6185 & 0.0862 & 0.1297 \\
\hline 8 & 0.620 & 0.613 & 0.440 & 0.612 & 0.217 & 0.254 & 0.6247 & 0.2730 & 0.2166 & 0.6131 & 0.0644 & 0.1031 \\
\hline 9 & 0.620 & 0.55 & 0.407 & 0.611 & 0.264 & 0.301 & 0.6271 & 0.2588 & 0.2098 & 0.6227 & 0.0598 & 0.0843 \\
\hline 10 & 0.600 & 0.234 & 0.250 & 0.615 & 0.132 & 0.190 & 0.6187 & 0.3187 & 0.2446 & 0.6167 & 0.0703 & 0.1074 \\
\hline 11 & 0.612 & 0.560 & 0.436 & 0.605 & 0.347 & 0.334 & 0.6222 & 0.2272 & 0.1767 & 0.5982 & 0.0863 & 0.1276 \\
\hline 12 & 0.611 & 0.433 & 0.401 & 0.600 & 0.333 & 0.313 & 0.6122 & 0.3593 & 0.2753 & 0.6252 & 0.0770 & 0.1123 \\
\hline 13 & 0.614 & 0.345 & 0.328 & 0.604 & 0.192 & 0.242 & 0.6200 & 0.2591 & 0.2067 & 0.6262 & 0.0630 & 0.0932 \\
\hline 14 & 0.625 & 0.509 & 0.400 & 0.616 & 0.166 & 0.221 & 0.6254 & 0.3543 & 0.2548 & 0.5841 & 0.0978 & 0.1514 \\
\hline 15 & 0.612 & 0.536 & 0.403 & 0.596 & 0.196 & 0.237 & 0.6238 & 0.3915 & 0.3142 & 0.6237 & 0.0389 & 0.0642 \\
\hline 16 & 0.617 & 0.408 & 0.374 & 0.617 & 0.216 & 0.265 & 0.6202 & 0.3179 & 0.2175 & 0.6064 & 0.1106 & 0.1596 \\
\hline 17 & 0.622 & 0.255 & 0.300 & 0.614 & 0.123 & 0.176 & 0.6257 & 0.3292 & 0.2336 & 0.6055 & 0.1215 & 0.1721 \\
\hline 18 & 0.612 & 0.533 & 0.433 & 0.600 & 0.206 & 0.258 & 0.6000 & 0.3369 & 0.2608 & 0.6000 & 0.1041 & 0.1541 \\
\hline 19 & 0.619 & 0.408 & 0.333 & 0.610 & 0.316 & 0.324 & 0.6161 & 0.2568 & 0.2139 & 0.6215 & 0.0728 & 0.1101 \\
\hline 20 & 0.605 & 0.345 & 0.290 & 0.588 & 0.224 & 0.255 & 0.6266 & 0.2713 & 0.2072 & 0.6259 & 0.0571 & 0.0899 \\
\hline 21 & 0.618 & 0.408 & 0.370 & 0.61 & 0.267 & 0.304 & & & & & & \\
\hline 22 & 0.623 & 0.500 & 0.411 & 0.610 & 0.176 & 0.230 & & & & & & \\
\hline 23 & 0.623 & 0.430 & 0.420 & 0.611 & 0.200 & 0.242 & & & & & & \\
\hline 24 & 0.613 & 0.600 & 0.430 & 0.586 & 0.256 & 0.265 & & & & & & \\
\hline 25 & 0.611 & 0.602 & 0.430 & 0.586 & 0.391 & 0.320 & & & & & & \\
\hline Mean & 0.615 & 0.501 & 0.400 & 0.600 & 0.256 & 0.273 & 0.6186 & 0.3317 & 0.2517 & 0.6139 & 0.0826 & 0.1234 \\
\hline SD & 0.006 & 0.108 & 0.055 & 0.009 & 0.083 & 0.046 & 0.0073 & 0.0762 & 0.0521 & 0.0110 & 0.0208 & 0.0283 \\
\hline
\end{tabular}

\section{DISCUSSION}

Grey level intensities of musculoskeletal US images are noisy and highly variable due to the nature of the ultrasound image and the musculoskeletal structure. As such, a fuzzy technique is an appropriate choice to represent it. The main contribution of this work is to detect only necessary details of the ultrasound image using a fuzzy edge detection method, which facilitates further applications, including geometric parameters evaluation. This approach offers a potential conduit through which the edge of the musculoskeletal ultrasound image can be stratified into three levels of edge information. The basic tenet of this approach is derived from a fuzzy inference method with carefully selected fuzzy parameters of membership function based on analysis of standard deviation. The third row of the table I shows two examples of the SD curve of the image after speckle noise reduction. According to this curve, parameters $\left(\mathrm{Cp}_{0}, \mathrm{Cp}_{1}, \mathrm{Cp}_{2}\right.$, and $\left.\mathrm{Cp}_{3}\right)$ were extracted. Detection of these parameters helped construct three membership functions. Analysis of the standard deviation of the thresholded images is a new approach for detecting parameters of fuzzy membership function. Edge detection was carried out using 12 fuzzy rules. This produces three levels of edge detection information. In all image samples, the images resulting from the $2^{\text {nd }}$ level showed more details because this level contained the highest value of
$\mathrm{SD}$, while images from the $1^{\text {st }}$ and $3^{\text {rd }}$ levels contained unnecessary image details (see two examples of experiment 1 and experiment 2 at the fifth row of the table I). At certain times, if edge detection of the ultrasound image included all details of image information, it was more difficult to perform the necessary calculations. For example, in the case of measuring the length of the muscle fascicle, if the edge detection image had all detail levels in one image, it was challenging to evaluate the length of the muscle fascicle automatically. Effectively, unnecessary information in the form of artifacts can mask the true edges.

To assess the fuzzy edge detection method performance, Pratt's FOM, Pinho and RBEM were utilised. The mean value of the recent assessment metric (RBEM) score for all samples was over 0.6. For Pratt's FOM and Pinho, the scores were 0.5 and 0.4 , respectively. In addition, there was a minimal difference in the RBEM score between all samples, and the standard deviation was approximately 0.006 (see Table II). In contrast with the traditional Canny edge detector method, Pratt's FOM and Pinho metrics were reported to be approximately 0.25 , while the standard deviation of RBEM was 0.009. Furthermore, it is difficult accurately evaluation the cross-sectional area and circumference of FPLT based on the resulting image from Canny edge detector. The main reason is this image included unnecessary information and some artifacts (see two examples 
of experiment 1 and experiment 2 at the sixth row of the table I). these examples shows images after applying Canny operator, extra details in these images including noise.

\section{CONCLUSIONS AND FUTURE WORK}

The novel fuzzy edge detection method was introduced in this work. This method can be used as a pre-processing step to automatically analyze musculoskeletal ultrasound images. Parameters of fuzzy membership functions were set up depending on the analysis of a standard deviation curve constructed from a set of thresholding images. The fuzzy edge detection method is more powerful than the Canny edge detector method because it can specify different levels of edge detection information with a robust score of quantitative assessment in all samples. Furthermore, it is an efficacious way for edge detection of Musculoskeletal Ultrasound Images because it is not time-consuming compared with other machine learning techniques, such as training CNNs.

We herewith suggest some possible avenues along which the performance of fuzzy edge detection method can be improved. To begin with, it is plausible that we could reconstruct the form of an edge as a large window in order to ameliorate performance. In addition, we would consider implementating this method on musculoskeletal ultrasound videos. Musculoskeletal ultrasound videos are a helpful tool in the illustration of the musculoskeletal system components across consecutive frames. It could provide useful support as a preprocessing step in some applications, such as optic flow methods [27], to calculate tendon length. Moreover, we would also consider implementation of the automated analysis on other biomedical images, such as Magnetic Resonance Imaging (MRI), biopsy images, and mammogram images. This could potentially expedite the full utilization of this approach and render it more applicable.

\section{ACKNOWLEDGMENT}

Many thanks to Ministry of Higher Education and Scientific Research in Iraq for funding this study. The authors would like to thank Kim Major, School of Health \& Rehabilitation, for her advice in using ultrasound machine.

\section{REFERENCES}

[1] Y. Li. Zhou, G. Zhou, and Y. Zheng, "Dynamic measurement of pennation angle of gastrocnemius muscles during contractions based on ultrasound imaging," Biomedical engineering online, 2012. doi: 10.1186/1475-925X11-63 pp.9-11, 2012.

[2] K. Chino, R. Akagi, and M. Dohi, "Measurement of muscle architecture concurrently with muscle hardness using ultrasound strain elastography," 55(7), PP.833-839, 2014.

[3] J. Canny, "A Computational Approach to Edge Detection,"IEEE Transactions on pattern analysis Machine Intelligence, 8(6), 679-698, 1986.

[4] L. Calatroni, Y. Van Gennip, C. B. Schönlieb, H. M. Rowland, and A. Flenner, "Graph Clustering, Variational Image Segmentation Methods and Hough Transform Scale Detection for Object Measurement in Images," J. Math. Imaging Vis., pp. 1-23, 2016.

[5] R. Gupta, I. Elamvazuthi, S. Dass, I. Faye, P.Vasant, J. George, \& F. Izzo, "Curvelet-based automatic segmentation of supraspinatus tendon from ultrasound image: a focused assistive diagnostic method,'BioMedical Engineering Online, 2014.

[6] S. I. Jabbar, C. R. Day, N. Heinz and E. K. Chadwick, "Using Convolutional Neural Network for edge detection in musculoskeletal ultrasound images," in 2016 IEEE International Joint Conference on Neural Networks (IJCNN), Vancouver, BC, 2016, pp. 4619-4626.

[7] W. Yang, Y. Chen, Y. Liu, L. Zhong, G. Qin, Z. Lu, Q. Feng, W. Chen, "Cas- cade of multi-scale convolutional neural networks for bone suppression of chest radiographs in gradient domain" Med. Image Anal. 35, 421-433, 2016, doi: 10.1016/j. media.2016.08.004.

[8] C. I. Gonzalez, P. Melin, J. R. Castro, O. Mendoza, and O. Castillo, “An improved sobel edge detection method based on generalized type-2 fuzzy logic," Soft Comput., pp. 773-784, 2014.

[9] C. Vanmathi, S. Prabu, "Image Steganography Using Fuzzy Logic and Chaotic for Large Payload and High Imperceptibility," International. Journal. Fuzzy System, vol. 20(2), pp. 460-473, 2018.

[10] R.C. Bueno, P.H.F. Masotti, J.F. Justo, D.A. Andrade, M.S. Rocha, W.M. Torres and R.N. de Mesquita, "Two-phase flow bubble detection method applied to natural circulation system using fuzzy image processing" Nuclear Engineering and Design, vol. 8(15), pp. 255-264, 2018.

[11] Rueda, S., Knight C., Papageorghiou A.T. and Noble J.A., 2015. Featurebased fuzzy connectedness segmentation of ultrasound images with an object completion step. Medical Image Analysis, 26(1), pp.30-46. Available at: http://dx.doi.org/10.1016/j.media.2015.07.002.

[12] M. Gonz, S. Massanet, A. Mir, and D. Ruiz-aguilera, "Edge Image Aggregation Method using Ordered Weighted Averaging Functions," in 2016 IEEE International Conference on Fuzzy Systems (FUZZ), 2016, pp. 1355-1362.

[13] AM. Badawi, AS. Derbala, and AM. Youssef "Fuzzy logic algorithm for quantitative tissue characterization of diffuse liver diseases from ultrasound images" International Journal of Medical Informatics 55(2), 135-147, 1999.

[14] K. Panetta, C. Gao, S. Agaian, and S. Nercessian, "A New ReferenceBased Edge Map Quality Measure," IEEE Trans. Syst. Man, Cybern. Syst., vol. 46, no. 11, pp. 1505-1517, 2016.

[15] C. Lopez-Molina, B. De Baets, and H. Bustince, "Quantitative error measures for edge detection," Pattern Recognit., vol. 46, no. 4, pp. 11251139,2013

[16] W. K. Pratt, Digital Image Processing, vol. 242. New York, NY, USA:Wiley, 1978.

[17] A. J. Pinho and L. B. Almeida, "Edge detection filters based on artificial neural networks," in Image Analysis and Processing. Berlin, Germany: Springer, 1995, pp. 159-164.

[18] S. C. Nercessian, S. S. Agaian, and K. A. Panetta, "A new reference- based measure for objective edge map evaluation," in Proc. SPIE Def. Security Sens. Conf., Orlando, FL, USA, 2009, Art. ID 73510J.

[19] H. R. Tizhoosh, G. Krell, and B. Michaelis, "On Fuzzy Enhancement of Megavoltage Images in Radiation Therapy," IEEE Conf. Fuzzy Syst. FUZZ-IEEE'97, vol. 3, pp. 1399-1404, 1997.

[20] M. Sonka, V. Hlavac and R. Boyle Image Processing Analysis and Machine Vision, $1^{\text {st }}$ edition, UK, Cambridge., 1993.

[21] M. Nachtegeal, D. Van der Weken, D. Van De Ville and E. Kerre "Fuzzy Filters for Image Processing", Germany, Springer-Verlag Berlin Heidelberg, 2010.

[22] T. Takagi and M. Sugeno, "Fuzzy identification of system and its applications to modelling and control," IEEE Transactions on Systems, Man, and Cybernetics, vol. SMC-15, no. 1. pp. 116-132, 1985.

[23] R. M. Haralick and S. R. Sternberg, "Image Analysis Morphology," no.4, pp. 532-550, 1987.

[24] S. Liu, J. Wei1, B. Feng, W. Lu1, B. Denby, Q. Fang and D. Jianwu, "An anisotropic diffusion filter for reducing speckle noise of ultrasound images based on separability," Proceedings of the 2012 Asia Pacific Signal and Information Processing Association Annual Summit and Conference, IEEE Hollywood, CA, 2012, pp. 1-4

[25] G. Grieg, O. Kubler, R. Kikinis, and F. A. Jolesz. "Nonlinear Anisotropic Filtering of MRI Data" IEEE Transactions on Medical Imaging, 11(2):221-232, June 1992

[26] P. Perona and J. Malik. Scale-Space and Edge Detection Using Anisotropic Diffusion. IEEE Transactions on Pattern Analysis and Machine Intelligence, 12(7):629-639, July 1990

[27] H. H. Nagel and W. Enkelmann, "An investigation of smoothness constraints for the estimation of displacement vector fields from image sequences." IEEE Trans. Pattern Analysis. Machine. Intelligence., vol. 8, no. 5, pp. 565-93, May 1986. 